\title{
Pregnancy vitamin D supplementation leads to greater offspring bone mineral density at age 4 years: findings from the MAVIDOS trial
}

\author{
E.M. Curtis ${ }^{1}$, R.J. Moon ${ }^{1,2}$, S. D’Angelo ${ }^{1}$, S.R. Crozier ${ }^{1}$, N.J. Bishop ${ }^{3}$, \\ J.S. Gopal-Kothandapani ${ }^{3}$, S.H. Kennedy ${ }^{4}$ A.T. Papageorghiou ${ }^{4}$, R. Fraser ${ }^{5}$, S.V. G. Gandhi ${ }^{5}$, \\ I. Schoenmakers, A. Prentice ${ }^{7}$, H.M. Inskip ${ }_{1,8}^{1,}$, K.M. Godfrey ${ }^{1,8}$, M.K. Javaid ${ }^{9,10}$, R. Eastell ${ }^{11}$, \\ C. Cooper ${ }^{1,8,10}$, N.C. Harvey ${ }^{1,8}$ and the MAVIDOS Trial Group \\ ${ }^{1}$ MRC Lifecourse Epidemiology Unit, University of Southampton, Southampton, UK, \\ ${ }^{2}$ Paediatric Endocrinology, University Hospitals Southampton NHS Foundation Trust, Southampton, UK, \\ ${ }^{3}$ Academic Unit of Child Health, Sheffield Children's Hospital, University of Sheffield, Sheffield, UK, \\ ${ }^{4}$ Nuffield Department of Women's \& Reproductive Health, John Radcliffe Hospital, University of Oxford, Oxford, UK, \\ ${ }^{5}$ Department of Obstetrics and Gynaecology, Sheffield Hospitals NHS Trust, University of Sheffield, Sheffield, UK, \\ ${ }^{6}$ Department of Medicine, Faculty of Medicine and Health Sciences, University of East Anglia, Norwich, UK, \\ ${ }^{7}$ MRC Nutrition and Bone Health, Clifford Allbutt Building, University of Cambridge, Cambridge, UK, \\ ${ }^{8}$ NIHR Southampton Biomedical Research Centre, University of Southampton and University Hospital Southampton \\ NHS Foundation Trust, Southampton, UK, \\ ${ }^{9}$ Nuffield Department of Orthopaedics, Rheumatology and Musculoskeletal Sciences, University of Oxford, Oxford, UK, \\ ${ }^{10}$ NIHR Oxford Biomedical Research Centre, University of Oxford, UK and \\ ${ }^{11}$ Department of Oncology and Metabolism, University of Sheffield, Sheffield, UK
}

In the multi-centre MAVIDOS trial, pregnancy vitamin D supplementation had a beneficial effect on neonatal bone mass amongst winter-born babies but not in other seasons ${ }^{(1)}$. We aimed to assess whether this effect on bone mineralisation is sustained into later childhood, with bone indices assessed at 4 years old in the Southampton participants. Demonstrating persistence of this effect into childhood would increase confidence in a long-term benefit of this intervention for life-long bone health.

In Southampton, Oxford and Sheffield, in a double-blind design, 1123 pregnant women with a baseline 25-hydroxyvitamin D level 25$100 \mathrm{nmol} / \mathrm{l}$ were randomised to $1000 \mathrm{IU} /$ day cholecalciferol or matched placebo from 14 weeks' gestation to delivery. At age 4 years (Southampton participants only, $\mathrm{n}=723$ births), offspring assessments included anthropometry and whole-body dual-energy $\mathrm{x}$-ray absorptiometry (DXA) [Hologic Horizon, yielding whole body less head (WBLH) bone mineral content (BMC), areal bone mineral density (aBMD), bone area (BA) and lean mass (LM)]. T-tests or Mann-Whitney U tests were used to compare bone DXA outcomes by maternal randomisation group. Linear regression was used to estimate the mean difference (represented by $\beta$ ) in outcomes between the two randomisation arms, adjusted for sex, and age at DXA. Outcomes were standardised to a standard deviation scale, for ease of comparison. Full ethics and MHRA approvals were granted. Trial registration: ISRCTN82927713, registered 11/04/2008.

$564 / 723(78.0 \%)$ children attended the 4-year visit; of whom 452 had a useable DXA with minimal movement artefact. Maternal pregnancy vitamin D supplementation led to greater offspring indices of bone mass compared with placebo, irrespective of season, for example WBLH aBMD at age 4 years, [supplemented group, 0.477 ( $95 \%$ CI: $0.472,0.481) \mathrm{g} / \mathrm{cm} 2$; placebo group $0.470(0.466,0.475)$ $\mathrm{g} / \mathrm{cm} 2 ; \beta=0.18(0.00,0.35) \mathrm{SD} \mathrm{p}=0.047$ ], and a trend towards associated greater LM [supplemented group, $9.25(9.08,9.42) \mathrm{kg}$; placebo group $9.01(8.83,9.18) \mathrm{kg} ; \beta=0.15(-0.02,0.31) \mathrm{SD} p=0.081]$. No effect was observed on fat mass. There was evidence of a larger effect of the intervention in the context of lower childhood calcium intake and lower physical activity. There was no difference in child weight, height or BMI between maternal randomisation groups.

This new evidence from a large placebo-controlled, double-blind randomised trial suggests that maternal pregnancy vitamin D supplementation leads to sustained improvement in offspring bone and muscle mass, informing public health approaches for the prevention of fractures.

We thank the MAVIDOS administrative staff, research nurses and participants for their contributions. MAVIDOS Trial Group: Nigel K Arden, Andrew Carr, Elaine M Dennison, Michael Clynes, Stephen J Woolford, M Zulf Mughal, David M Reid. EMC, RJM and SD are joint first authors; $\mathrm{CC}$ and $\mathrm{NCH}$ are joint senior authors. This work was supported by grants from Arthritis Research UK 17702, Medical Research Council (MRC \#405050259; \#U105960371), Bupa Foundation, NIHR Southampton Biomedical Research Centre, University of Southampton and University Hospital Southampton, and NIHR Oxford Biomedical Research Centre, University of Oxford. EC was supported by the Wellcome Trust (\#201268/Z/16/Z). The work leading to these results was supported by the UK Royal Osteoporosis Society Osteoporosis and Bone Research Academy, the European Union's Seventh Framework Programme (FP7/2007-2013), projects EarlyNutrition and ODIN under grant agreements numbers 289346 and 613977, and by the BBSRC (HDHL-Biomarkers, BB/ P028179/1), as part of the ALPHABET project, supported by an award made through the ERA-Net on Biomarkers for Nutrition and Health (ERA HDHL), Horizon 2020 grant agreement number 696295. We are extremely grateful to Merck GmbH for the kind provision of the Vigantoletten supplement. Merck GmbH had no role in the trial execution, data collection, analysis or manuscript preparation.

\section{Reference}

1. Cooper C, Harvey NC, Bishop N, et al. (2016) Lancet Diabetes Endocrinol 4(5), 393-402. 